\title{
Research on Energy Saving Technology of Central Heating in Venture Towns
}

\author{
Chang Qu ${ }^{1, a}$ \\ ${ }^{1}$ Mine Area Service Department, Daqing Oilfield of CNPC, Daqing, 163000, China
}

Keywords: Sub-period adjusting heat, Optocoupler isolation, No power consumption, Climate compensation

\begin{abstract}
This is in response to the Eighteen big call to implement national planning policy in 12th Five-Year, 12th Five-Year in Daqing city planning in the premise of improving heating quality under the goal of saving energy, Daqing area planning focused on the implementation of energy-saving heating system is in line with national policy requirements, timely is important. Five years of special planning to improve the quality of heating and energy conservation, not only meets the policy requirements of the national level, but also conforms to the planning requirements of the local level in Daqing.
\end{abstract}

\section{Intelligent Temperature Control Technology of Climate Compensation}

According to the city area business hot line in the heating period, according to the numerical weather prediction day average temperature finally calculate heat load, at the same time, the peak in the mid-term operation need to increase some allowance, to meet the load demand at night. In the daytime, the outdoor temperature is higher, which results in the waste of heat energy. In order to save energy and improve the means of control, a new control idea is put forward by studying the curve. For outdoor air temperature within 24 hours of operation, the outdoor maximum temperature is - 1 degrees $\mathrm{C}$, and the minimum temperature is -11 DEG C. According to the usual load calculation method, the outdoor average temperature is -8 DEG C. Judging from the outdoor temperature curve, it is not reasonable to adopt an invariable load operation mode. Through the study of curves, a new control idea is put forward, which is divided into different periods of 24 hours, and each period of time is operated according to the average temperature of the period, so as to achieve the purpose of energy saving.

\section{Modification Design}

Central heating system is composed of three main parts: heat source, heat supply network and heat user. It is a complicated system. Heat medium will produce energy loss in the process of production, transportation and use, resulting in waste of energy. In the production process of heat medium, the boiler efficiency determines the utilization of energy, the cause of the low efficiency of boiler: boiler heat capacity is small; the operation efficiency to full load operation and low combustion efficiency; low combustion caused by inadequate fuel. The energy loss of heat medium in the conveying process is mainly due to heat dissipation along the pipeline; pipeline leakage phenomenon; pipe network hydraulic imbalance caused by the uneven; the unreasonable operation mode and replenishment mode. In the process of using heat media, the waste is the room temperature cannot be adjusted; window cooling; energy saving consciousness is not strong and so on. By adding an electric regulating valve on the bypass pipe of the two-stage network plate heat exchanger, the quality and regulation are achieved. The output input signal is optically isolated, and in the command center, the upper computer can realize remote manual or automatic adjustment. According to the area of traditional charging system has many problems, user awareness is not strong and will open the window cooling, heating metering and charging can promote energy saving has become a conscious behavior of people using heat, but the unit building in China is not suitable for household heat 
metering, when users to turn off or close the bulkhead valve when the temperature difference between two between the big, caused by heat transfer cannot be ignored, the increase of household heat heating consumption increases, there is no good way to solve. In each building before the installation of heat metering installation, heat distribution meter inside each radiator, so as to enhance people's awareness of energy conservation, promote energy saving, but also can effectively promote energy-saving building envelope in China.

\section{Selecting Types of Main Equipment}

The main use of the original valve, a set of automatic electronic actuator relates to the main body part of the increase in the spirit, can use original equipment on the use of the original equipment, avoid duplication of investment, the funds used where it is most needed. And the original ordinary butterfly valve into manual electric butterfly valve, to achieve automatic adjustment function. The fluid transmission and distribution network knowledge shows that the heating network can be divided into branch network and loop network, the difference between the two is that the pipe flow is determined. Branch network has the advantages of simple structure, less economic costs, but the heating stability is gradually being replaced by circular pipe, pipe network in addition to heating stability but also has the advantages of energy saving, can realize the economic operation. In the multi heat source heating network, according to the change of external conditions to flexibly adjust the source type, preferred source heating of low energy consumption, environmental protection, good economy, with a hot heat source in the peak of the help of high consumption, so as to save energy and realize the economic operation of heating. The reliability of the multi heat source network heating system is good, and when one of the heat sources fails, the other heat sources can be continued to heat the users, without affecting the heating effect.

For the new station within the equipment, to achieve the following points:

(1) receive 4 20mA DC. current signal control. Feedback 4 20mA DC. current signal (valve opening).

(2) intelligent positioning, high accuracy, good damping characteristics.

(3) the body surface should have buttons, you can adjust the opening by hand, but also to display the opening of the electronic plate and valve position feedback value.

(4) the site is equipped with hand wheels, with manual and automatic switching facilities.

(5) output stroke characteristic transformation: we require that the characteristics are continuous, and there is correlation and valve position.

(6) the action mode set in the actuator is required to be performed.

(7) when the input signal is interrupted, the "interrupt" mode setting is required in the actuator.

(8) IP55 is the protection level of the executing agency.

(9) dead zone within $0.5 \% \mathrm{FS}$.

(10) linearity within $0.6 \% \mathrm{FS}$.

(11) there are protective devices such as open and close limit switches and overheat protection.

\section{Software Design}

Boiler control room according to the Daqing Meteorological Station forecast outdoor maximum and minimum temperature, the computer system automatically calculates the time period of the outdoor temperature operating curve and the 24 hours all day long is divided into four stages, the control program according to the sunshine, wind speed and wind direction are automatically calculated for each different time period the average outdoor temperature and the corresponding heating temperature and take different heating parameters, high and low down, to ensure that residents of normal temperature, saving heat. The combustion efficiency of the boiler not only with fuel, is also related to the water temperature of the boiler, fuel combustion more fully, the higher the temperature of the water boiler efficiency is high, improve the efficiency of the boiler is to enhance combustion and improve water temperature. The specific measures are: using water mixing device, 
ensure the boiler long time full load operation. According to the change of user end of the heat load, the water mixing device out of reasonable mixing ratio of the boiler water temperature remained high, and ensure the efficient operation of the boiler. The high heat conductivity of the heat pipe makes the heat pipe economizer greatly improve the thermal efficiency of the boiler. Small and medium sized boiler mixed burning cinder by reduction of carbon content in slag, improve the combustion stability of boiler, some studies show that when the coal and slag ratio is about four to one. The boiler is equipped with a layered coal feeder, and a stratified combustion technology is adopted to improve the combustion environment of the boiler and increase the fuel combustion efficiency of the boiler.

\section{Key Technologies and Innovations}

The main use of the original valve, a set of automatic electronic actuator relates to the main body part of the increase in the spirit, can use original equipment on the use of the original equipment, avoid duplication of investment, the funds used where it is most needed. And the original ordinary butterfly valve into manual / electric butterfly valve to achieve automatic adjustment function.

The boiler main control room receives the latest online average temperature forecast, computer control software will run automatically after heating operation given different time curves, and all day long 24 hours is divided into four stages, the control program according to the sunshine, wind speed and wind direction are automatically calculated for each different time period the average outdoor temperature and the corresponding the heating temperature, adopt different heating parameters, high and low down, to ensure that residents of normal temperature, saving heat. Hydraulic balance valve is installed to improve the hydraulic maladjustment and thermal maladjustment of the pipe network. Because the heating pipe of the large, difficult to achieve hydraulic balance real, even if the calculation accuracy is difficult to guarantee the balance of resistance in the opening and operation adjusting right to open the valve, to achieve the balance between users. The hydraulic balance valve is operated flow control valve and self-operated pressure control valve, regulating principle of both are similar, do not need external power, only need to change depending on the resistance of fluid flow or pressure difference control valve opening. The scope of application is also different, self-flow control valve for the temperature control valve is not installed in the constant flow system, and self-pressure differential valve is suitable for the installation of temperature control valve variable flow system.

Improve the buried technology of the two-pipeline network, strengthen the insulation of the pipe network, and reduce the heat loss during the transportation process. Strengthen the pipeline insulation work, can reduce heat loss in the transportation process, and can effectively reduce the pipeline leakage phenomenon, effectively reduce the heat loss of the two-level network, a data show that the heat efficiency of the buried pipeline is greater than $90 \%-95 \%$. Change the water supply mode of the pipe network to reduce the water recharge. Large water recharge rate is a waste of energy in the two-level pipe network and thermal power station. In addition to minimizing leakage, reducing unnecessary water points to reduce water recharge rate.

Under normal circumstances, by adjusting the self-operated differential pressure valve for a water pipe network to the two-network supplement; if a pipe network pressure is not enough or the system of water loss is large, by adjusting the solenoid valve to the two-level pipeline replenishment; only in the accident and flushing pressure test cases from the water replenishing tank pumping water to the system via a water pump. Change the operation regulation mode of pipe network, replace the original quality regulation constant flow system with variable flow system. The traditional method of constant flow quality control operation, the change of heat source temperature needs several hours or even more than ten hours to influence the users. It not only wastes energy, but also cannot meet people's comfort requirements. The system of pumps to variable speed pump, such changes can flow adjusted according to the change of load, achieve the purpose of saving energy by frequency conversion, according to the relevant data show that the heating system with frequency conversion technology and energy saving rate can reach more than $30 \%$. 


\section{Prediction of Economic Returns}

According to the 2016-2017 heating operation, operation of mid peak accounted for $70 \%$ of the total run time, through the regulation of temperature should be reduced to $0.6 * 0.7=0.42$ DEG C, for the central heating system of entrepreneurial city, the outdoor temperature changes every 1 degrees for the variation of system load maximum load design $=72 /(18+26)=1.636 \mathrm{~W} / \mathrm{m} 2$, equal to $0.025 \mathrm{GJ} / \mathrm{m} 2$. When we adopt the time interval heating regulation, we can save $0.025 \mathrm{GJ} / \mathrm{m} 2 \times 0.42$ $\times 420 \times 30 / G J=132.3$. By means of climate compensation and intelligent temperature control technology, the two-level network can be adjusted remotely, manually or automatically. It has good energy saving potential, and can effectively reduce the labor intensity of workers and improve the management level of production and operation.

\section{Existing Problems and Solutions}

As the outdoor temperature is the outdoor maximum and minimum temperature according to the weather forecast, the operating curve of each time section calculated by the computer system automatically has some errors, which affect the adjustment. The heat exchange station of incomplete data, network operating parameters is not accurate enough, it is difficult to achieve quantitative management. There is no good combination of computer automatic control system, when the pipe network fails, problems cannot be found in time, affecting the reliability of network operation and waste of energy. Domestic experts on the calculation of household heat metering dispute. Aware of the defects of China's welfare heating system, China began to implement the reform of learning in Europe and the United States heating household metering system, but did not achieve a good energy saving effect, the household metering system is not wrong, but cannot copy the calculation method of the advanced countries, should be combined with the characteristics of unit building of our country to find the calculation method suitable for our country, to achieve household heat metering. Set up a complete set of outdoor temperature measurement device, real-time monitoring of the external temperature, thereby reducing the adjustment error.

\section{Conclusions}

The application and implementation of the project, improve the original network control and regulation system, realizes the remote two-level network control the temperature in the boiler owner the host computer. It can achieve the purpose of continuously and timely control of heat load at a different time of day.

\section{References}

[1] Zhang Lei, Han Meng, Lu Xiaoqian. Analysis on Coal Consumption and Potential Energy- saving of Central Heating in Northern Provinces of China Under Urbanization Background [J]. CHINA Population, Resources and Environment, 2015, 25(8): 58-68.

[2] Zhang Dandan, Di Yuhui, Huang Yinpeng. The Development of Central Heating Energy-saving Technology [J]. Contamination Control \& Air-Conditioning Technology, 2016(3): 57-60.

[3] He Lei. Research on the Energy Saving Operation of the Central Heating Heat Exchange Station [J]. Engineering and Technological Research, 2016(5): 83+117.

[4] Wang Yang, Tian Guansan, Zhang Mingguang. Optimizaiton and operation analysis of heating converting satation of central heating system [J]. Jouranl of Shandong Jianzhu University, 2009, 24(6): 574-580. 